\title{
The SHiP experiment and its detector for neutrino physics
}

\author{
Annarita Buonaura*t \\ Universitá di Napoli Federico II e INFN Napoli \\ E-mail: annarita.buonauraena.infn.it
}

\begin{abstract}
SHIP is a new general purpose fixed target facility, proposed at the CERN SPS accelerator. In its initial phase the $400 \mathrm{GeV}$ proton beam will be dumped on a heavy target with the aim of integrating $2 \times 10^{20}$ pot in 5 years. A detector downstream of the target will allow to search long-lived exotic particles with masses below $\mathrm{O}(10) \mathrm{GeV} / \mathrm{c}^{2}$ forseen in extension of the Standard Model. Another dedicated detector, that will be the focus of this talk, will allow to study active neutrino crosssections and angular distributions. The neutrino detector consists of an emulsion target, based on the Emulsion Cloud Chamber technology fruitfully employed in the OPERA experiment. The Emulsion Cloud Chamber will be placed in a magnetic field, with the so-called Compact Emulsion spectrometer, a few $\mathrm{cm}$ thick chamber for the charge and momentum measurement of hadrons. This will provide the leptonic number measurement also in the hadronic tau decay channels. The detector will be hybrid, using nuclear emulsions and electronic detectors for the time stamp of the events and the measurement of the muon momentum. The muon system will also be based on the design of the one used in the OPERA experiment.
\end{abstract}

XXIII International Workshop on Deep-Inelastic Scattering,

27 April - May 12015

Dallas, Texas

\footnotetext{
* Speaker.

On behalf of the SHiP Collaboration.
} 


\section{Introduction}

The 2012 was the year of the success of the Standard Model (SM). Indeed, the discovery of the Higgs boson [1],[2] was a milestone in the field of Particle Physics. It provided a further validation of the accuracy of the SM in describing Nature's fundamental constituents and their interactions. However, there are still some phenomena that the SM is unable to explain: the existence of dark matter (DM) and its nature, the baryonic asymmetry of the Universe and neutrino masses. They all hint at the existence of new physics and of particles either too heavy or too weakly interacting to be detected but that could represent the key to work out a solution to the previous listed issues.

So far, new physics has been searched at LHC, looking for high mass particles, with ordinary couplings to matter, at always increasing collision energies.

A physics program focusing on very weakly couplings- hence complementary to that of LHCmight on the other hand be supplied by a beam dump facility. It is in this framework that the SHiP experiment (Search for Hidden Particles, [4], [5]) sets. It will look for very weakly interacting non-SM particles with masses in the $\mathrm{GeV}$ region, collectively belonging to the so called "Hidden Sector" (HS), such as: light sgoldstinos that appear in the breaking of symmetry of the SUSY theory, sterile neutrinos called Heavy Neutral Leptons (HNL) also foreseen in a minimal extension ( $v$ MSM [3]) of the SM as heavy (order of GeV) right handed partners of the SM neutrinos and other particles singlets with respect to the SM.

Thanks to the peculiar properties of the facility, the SHiP experiment can also be considered a SM neutrino factory, in particular of tau neutrinos. Hence, it will host a neutrino detector to discover the tau anti-neutrino and to study tau neutrino and anti-neutrino cross-sections.

\section{A detector to reveal the Hidden Sector}

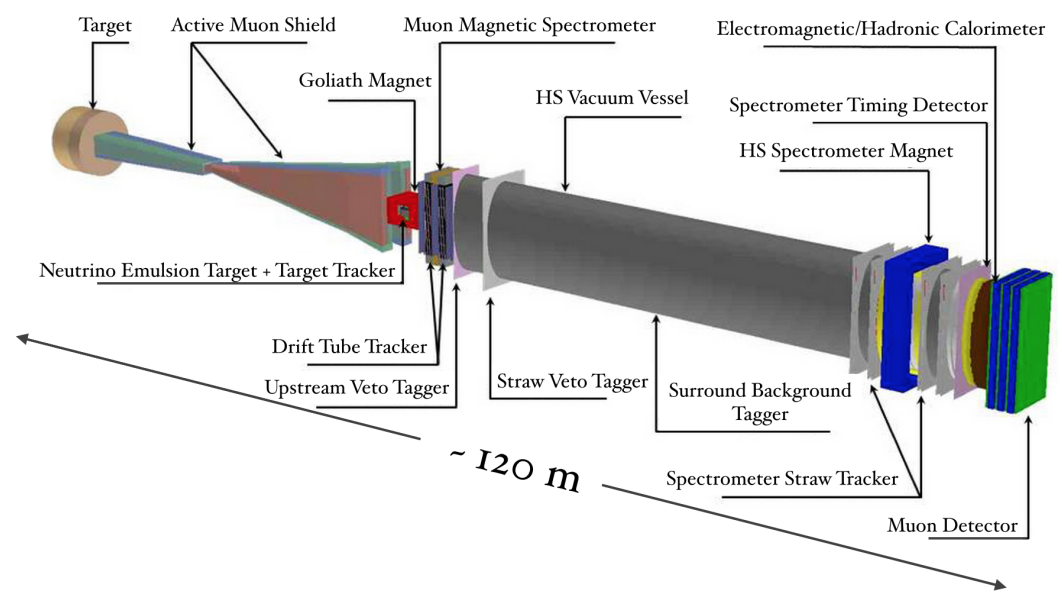

Figure 1: Overall detector layout of the SHiP facility.

The overall detector layout is shown in fig.1. It is made of a high density proton target, followed by a hadron stopper and a muon shield. A neutrino detector (described in section 3.1) and a $50 \mathrm{~m}$ long evacuated decay volume are located downstream. The HS detector concept accomplishes the task of reconstructing HS particle decays in all their possible final states. HS particles 
or, shortly, Hidden Particles (HP) are produced through hadron decays $(\pi, K, D, B, \ldots)$ and are supposed to decay in SM particles. In tab. 1, the different decay modes of the HP-according to the theoretical model considered-are reported. It is thus of crucial importance to perform a full reconstruction of the event, as well as particle identification, in order to be less model dependent. A big challenge is also represented by the background rejection being both production and decay rates of the HP extremely small due to the very weak couplings expected.

\begin{tabular}{cc}
\hline Models & Final states \\
\hline Neutrino portal, SUSY neutralino & $l^{ \pm} \pi^{\mp}, l^{ \pm} K^{\mp}, l^{ \pm} \rho^{\mp}, \rho^{ \pm} \rightarrow \pi^{ \pm} \pi^{0}$ \\
Vector, scalar, axion portals, SUSY sgoldstino & $l^{+} l^{-}$ \\
Vector, scalar, axion portals, SUSY sgoldstino & $\pi^{+} \pi^{-}, K^{+} K^{-}$ \\
Neutrino portal, SUSY neutralino, axino & $l^{+} l^{-} v$ \\
Axion portal, SUSY sgoldstino & $\gamma \gamma$ \\
SUSY sgoldstino & $\pi^{0} \pi^{0}$ \\
\hline
\end{tabular}

Table 1: Summary of the main decay modes of hidden particles in various models $(l=e, \mu)$

\subsection{Experimental requirements and the apparatus}

SHiP searches HP produced in heavy flavour hadron decays (fig.2a). The choice of a proton beam with an energy of $400 \mathrm{GeV} / \mathrm{c}(\sqrt{s}=27 \mathrm{GeV} / \mathrm{c})$ is the optimal solution because at this energy the production cross sections of $c \bar{c}$ tend to saturate (fig.2b) [6], [7], [8].

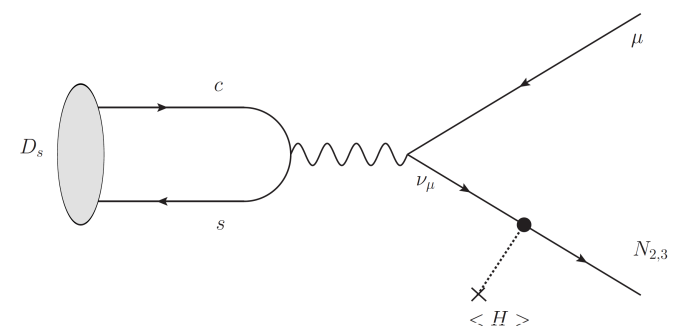

(a) Example of a charmed hadron decay producing Heavy Neutral Lepton.

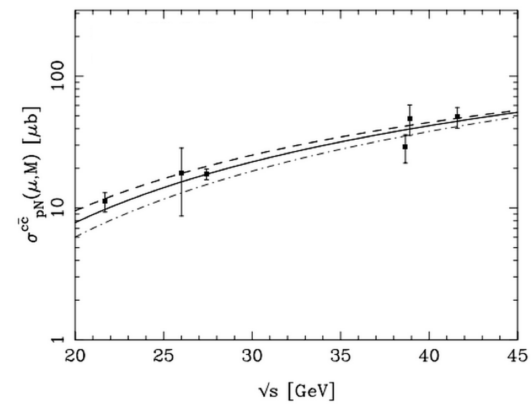

(b) Total c $\bar{c}$ production cross sections at fixed target energies [6], [7], [8].

Figure 2: Example of a production mechanism of HNLs and $c \bar{c}$ cross-section for different $\sqrt{s}$

A hybrid target made of blocks of molybdenum and tungsten, materials with a short interaction length, is adopted to maximize neutrinos from charmed hadrons while minimizing those coming from pions and kaons decays. The length of the target is chosen to contain possible hadronic showers with minimum leakage, but in order to have a greater efficiency a hadron stopper made of $5 \mathrm{~m}$ of Iron is placed downstream of the target.

HP produced in the decay of charmed hadrons show a significant transverse momentum, hence requiring, to maximize the acceptance, to place the decay volume as close as possible to the proton 
target. Furthermore, due to the smallness of the couplings of HP to SM particles, the decay vessel has to be $50 \mathrm{~m}$ long to be able to detect the decay products of these very long-lived particles (the typical lifetimes are larger than $10 \mu \mathrm{s}$, with corresponding decay distances of the order of the $\mathrm{km}$ ). This task is performed using a magnetic spectrometer, calorimeters and muon detectors placed at the far end of the decay volume.

As already pointed out, it is essential to design the beam line in such a way the the background sources are reduced. The main source of background is the muon flux coming from short-lived resonances and from the decay of residual pions and kaons. The reduction of this type of background is also of great advantage for the neutrino physics program. Indeed, since the target is made of nuclear emulsions, precise limits on the acceptable muon rates are set.To clear a $5 \mathrm{~m}$ horizontally wide region, a $48 \mathrm{~m}$ long active muon shield based on magnetic deflection of the muons in the horizontal plane is introduced.

On the other hand, to reduce the combinatorial background from the residual muons entering the decay volume and muons deflecting off the cavern walls, the proton spills are prepared with a slow (1s) and very uniform beam extraction.

Active neutrinos are a potential background to HP detection. While neutrino interactions inside the decay volume can be considered negligible, being the vessel kept at low pressure $\left(10^{-6} \div 10^{-5}\right.$ bar $)$, it is important to provide efficient protection against neutral particles produced in charged-current (CC) and neutral-current (NC) neutrino interactions in the material upstream of the fiducial volume. For this reason, a combination of light taggers must be located upstream and at the beginning of the fiducial volume.

\section{The neutrino detector}

With a proton energy enhancing the production of charmed mesons and in particular of $D_{s}$ mesons, a high flux of $v_{\tau}$ and $\bar{v}_{\tau}$, together with neutrinos of all flavours, is expected.

Tau neutrino is still the less known particle of the SM. Since its discovery in 2000 by the DONUT experiment with the observation of 9 candidate events out of a background of 1.5 , only other 5 events of tau neutrinos have been detected so far during the search of $v_{\mu} \rightarrow v_{\tau}$ oscillation exploited by the OPERA experiment [9], [10], [11], [12], [13], allowing to assess the discovery of $v_{\mu} \rightarrow v_{\tau}$ oscillations in appearance mode with a significance larger than $5 \sigma$ [14]. On the other hand, no $\bar{v}_{\tau}$ has ever been detected yet, making it the last missing tile of the SM.

The neutrino detector (fig.3a) designed for the SHiP experiment will thus be able to perform the first direct observation of the $\bar{v}_{\tau}$ and, thanks to the expected unprecedented statistics of tau neutrinos and anti-neutrinos, it will be able to study the properties and the cross-section of $v_{\tau}$ and $\bar{v}_{\tau}$.

\subsection{Experimental requirements and the apparatus}

The tau neutrino detector is located in between the active muon shield and the decay vessel. It is made of a neutrino target region followed by a muon magnetic spectrometer (fig.3a).

The neutrino target has a mass of $\sim 9.6$ tons and a modular structure. The unit is a "brick" with a Compact Emulsion Spectrometer (CES) as shown in fig.3b. It is placed in a magnet that provides a vertical magnetic field that ranges from 1 to $1.5 \mathrm{~T}$ in the target region. 


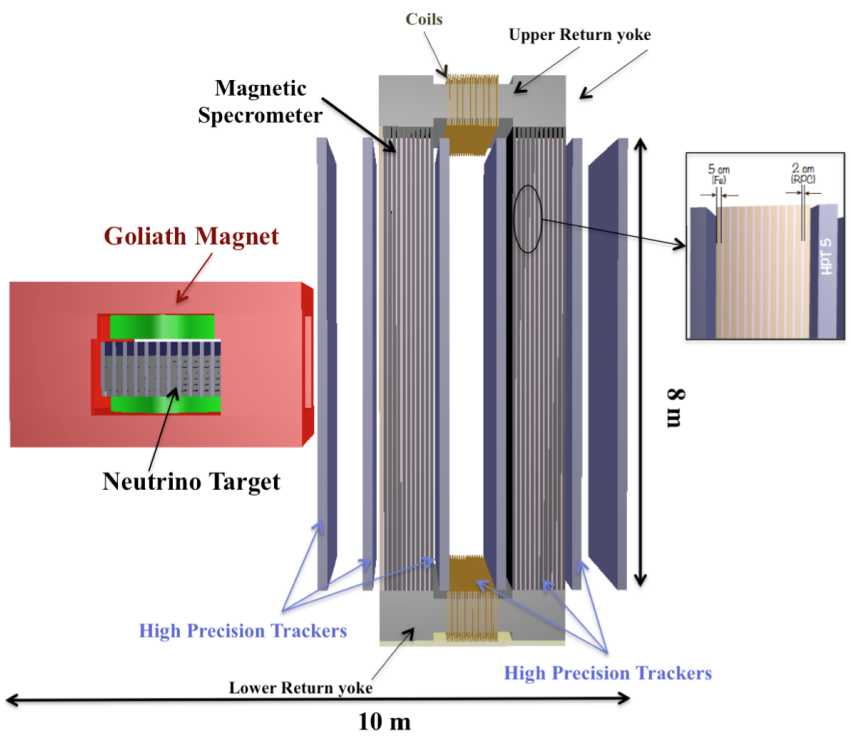

(a) Layout of the neutrino detector.

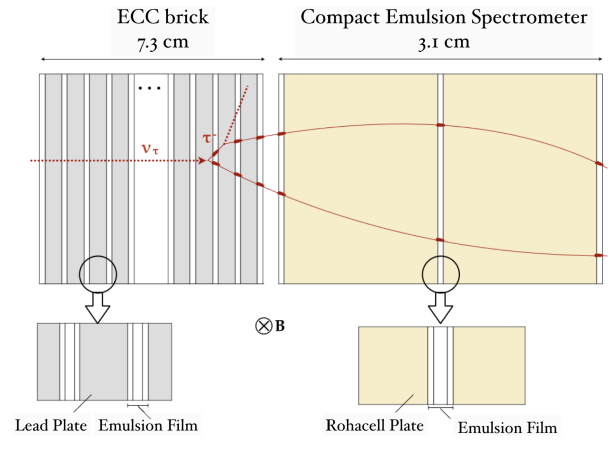

(b) Schematic representation of the neutrino detector unitary cell.

Figure 3: View of the tau neutrino detector and of its fundamental unit.

The brick employs the Emulsion Cloud Chamber (ECC) technology interleaving $1 \mathrm{~mm}$ lead plates serving as passive material and $300 \mu \mathrm{m}$ emulsion films serving as tracking devices with micrometric resolution, for a total thickness of $\sim 7 \mathrm{~cm}$. This technology has been largely exploited by the OPERA experiment and identifies $v_{\tau}$ on topological basis, exploiting the micrometric resolution to disentangle the $\tau$ lepton production and decay vertices. Further kinematical cuts are then applied to the candidate events in order to discard background processes.

The CES - attached downstream of each ECC brick-is needed to measure the electric charge an momentum of the $\tau$ daughters for the $v_{\tau}$ and anti- $v_{\tau}$ identification. It is thus made of a sandwich of light material plates (Rohacell) and emulsion films for a total length of $3.1 \mathrm{~cm}$. It is designed to be able to perform charge measurement for hadrons with momentum up to $10 \div 12 \mathrm{GeV}$ through the sagitta method.

The neutrino detector unitary cells are arranged in 11 vertical walls, each made of 105 of these units, as shown in fig.4. Each wall is interleaved with target trackers. As a matter of fact, electronic detectors are needed to provide the time stamp of the neutrino interaction occurred in the brick and to link the muon tracks in the target with the magnetic spectrometer. Different options are considered as electronic trackers: scintillating fibres or micro-pattern gas detectors like GEM or micromegas. The physics performances needed in a $1 \mathrm{~T}$ field are $100 \mu \mathrm{m}$ position resolution on both coordinates to disentangle tracks from the same interaction coming from 2 different vertices and high efficiency for angles up to $1 \mathrm{rad}$.

The muon magnetic spectrometer has the same structure of the OPERA magnetic spectrometer. It is made of 2 arms, each one made of 12 iron layers, $5 \mathrm{~cm}$ thick, interleaved with $11 \mathrm{RPC}$ planes, $2 \mathrm{~cm}$ thick, and it is also equipped with 6 drift tube tracker planes. It is of fundamental importance to identify the muons produced in neutrino interactions and in $\tau$ decays and measure their charge and momentum. As a matter of fact, muon identification at the neutrino interaction vertex is 


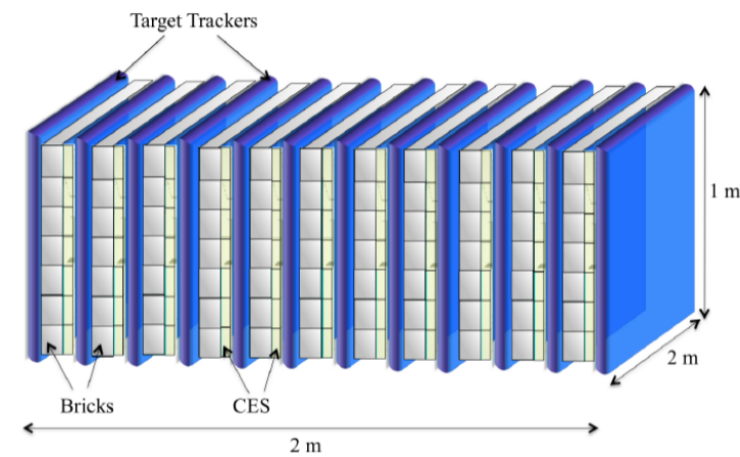

Figure 4: Schematic view of the target with the 12 target tracker layers.

crucial for background rejection. The main background to the $\tau$ lepton identification comes from the decay of charmed hadrons produced in $v_{\mu}$ CC interactions, that exhibit the same decay topology, if the primary lepton is not identified. The direction of the $1.5 \mathrm{~T}$ magnetic field is opposite in the two arms. This allows to have a large acceptance an efficiency of more than $99 \%$ in charge identification and to have a momentum resolution better than $25 \%$.

\subsection{Physics with the neutrino detector}

Tau neutrinos are copiously produced via $D_{s} \rightarrow \tau \nu_{\tau}$ and subsequent $\tau \rightarrow v_{\tau}$ decay. In five years run, $5.7 \times 10^{15} v_{\tau}+\bar{v}_{\tau}$ per $2 \times 10^{20}$ protons on target are expected. A high rate of $v_{e}$ and $v_{\mu}$, induced by the decay of soft pions and kaons, is also foreseen. The spectra and the neutrino yield at the beam dump are reported in fig.5a and in the left colum of tab.2, respectively. The expected number of CC deep-inelastic neutrino interactions during the whole data taking is reported in the right column of Tab.2 while the energy spectra are shown in fig.5c. All the previous results have been obtained thanks to a Geant 4 based simulation of the facility. It is important to notice that almost $7000 v_{\tau}$ and $3500 \bar{v}_{\tau}$ charged current interactions are expected, a statistics sufficient to perform cross-section studies.

\begin{tabular}{c|cc|cc}
\hline & $<$ E $>$ & Beam dump & $<$ E $>$ & CC DIS \\
\hline$N_{v_{\mu}}$ & 1.4 & $4.4 \times 10^{18}$ & 29 & $1.7 \times 10^{6}$ \\
$N_{v_{e}}$ & 3 & $2.1 \times 10^{17}$ & 46 & $2.5 \times 10^{5}$ \\
$N_{v_{\tau}}$ & 9 & $2.8 \times 10^{15}$ & 59 & $6.7 \times 10^{3}$ \\
$N_{\bar{v}_{\mu}}$ & 1.5 & $2.8 \times 10^{18}$ & 28 & $6.7 \times 10^{5}$ \\
$N_{\bar{v}_{e}}$ & 4 & $1.6 \times 10^{17}$ & 46 & $9.0 \times 10^{4}$ \\
$N_{\bar{v}_{\tau}}$ & 8 & $2.8 \times 10^{15}$ & 58 & $3.4 \times 10^{3}$ \\
\hline
\end{tabular}

Table 2: Integrated neutrino yield for $2 \times 10^{20}$ p.o.t. for the different neutrino flavors: at the beam dump (left) and CC DIS interactions (right). Energies are in GeV.

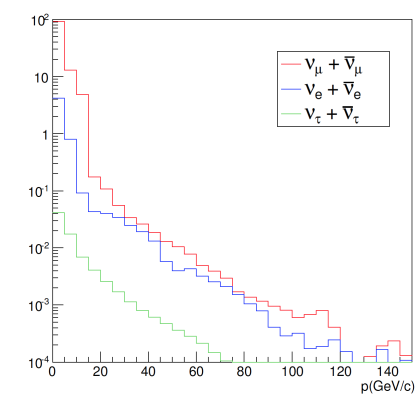

(a) Beam dump.

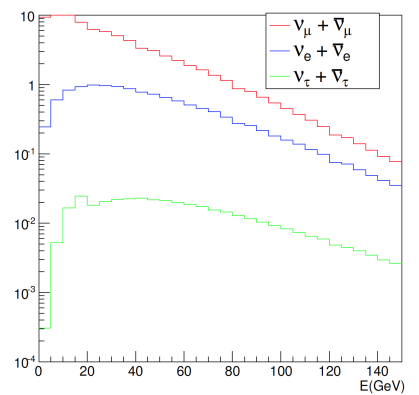

(b) CC interacting.
Figure 5: Energy spectra of the three neutrino flavors at the beam dump (a) and of CC interactions in the neutrino target(c). The total number of neutrinos is normalized to 100. 
From the study of the deep-inelastic scattering (DIS) cross-section of $v_{\tau}$ on nucleons, it is possible to extract the $F_{4}$ and $F_{5}$ structure functions, which are negligible in muon (and electron) neutrino interactions because of a suppression factor proportional to the square of the charged lepton mass. At leading order, in the limit of massless quarks and target hadrons, $F_{4}=0$ and $2 x F_{5}=$ $F_{2}$, where $x$ is the Bjorken- $x$ variable [15]. Calculations at NLO show that the $F_{4}$ contribution to this cross-section is about $1 \%$ [16].

Thanks to the large flux of $v_{e}$ and $v_{\mu}$ reaching and interacting in the neutrino target not only studies on $v_{\tau}$ physics can be performed. As a matter of fact, the strange-quark content of the nucleon can be measured by means of the charmed hadron production in anti-neutrino interactions. In 5 years run, more than $10^{5}$ neutrino induced charmed hadrons are expected. The advantage of using a detector with the micrometric resolution allows to identify these hadrons through the decay topology. Not only di-lepton events have thus to be taken into account. Hence, the statistics available exceeds by more than one order of magnitude the one available in previous experiments. A simulated SHiP data sample normalized to the expected statistics was used to estimate the distribution of $v_{\mu}\left(\bar{v}_{\mu}\right)$ interactions with charm production. The potential impact of simulated charm data was assessed by adding them to the NNPDF3.0 NNLO fit [17]. Defining $s^{ \pm}$as $s^{ \pm}=s(x) \pm \bar{s}(x)$, almost a factor two gain on the accuracy is achieved in the $x$ range between 0.03 and 0.35 for $s^{+}$and in the one between 0.08 and 0.30 for $s^{-}$.

\section{References}

[1] ATLAS Collaboration, Phys.Lett. B716 (2012) 129.

[2] CMS Collaboration, Phys.Lett. B716 (2012) 3061.

[3] T.Asaka, M.Shaposhnikov, PL B620 (2005) 17.

[4] SHIP Collaboration, CERN-SPSC-2015-016, SPSC-P-350, arXiv:1504.04956 [physics.ins-det], (2015).

[5] SHIP Collaboration, CERN-SPSC-2015-017, arXiv:1504.04855 [hep-ph], (2015).

[6] C. Lourenco and H. Wohri, Phys.Rept. 433, 127-180, (2006).

[7] H. Abramowicz et al., JHEP. 1309, 058, (2013).

[8] K. Olive et al., Review of Particle Physics, Chin.Phys. C38, 090001, (2014).

[9] OPERA Collaboration, JINST 4 (2009) P04018.

[10] OPERA Collaboration, Phys.Lett. B691 (2010) 138-145.

[11] OPERA Collaboration, JHEP 11 (2013) 036.

[12] OPERA Collaboration, Phys. Rev. D89 (2014) 051102.

[13] OPERA Collaboration, PTEP 2014 (2014) $10101 \mathrm{C} 01$.

[14] OPERA Collaboration, arXiv:1507.01417 [hep-ex], (2015)

[15] C. H. Albright and C. Jarlskog, Nucl. Phys. B 84 (1975) 467.

[16] M. H. Reno, Phys. Rev. D 74 (2006) 033001.

[17] R. D. Ball et al., Parton distributions for the LHC Run II. (2014). 